Meta

Journal des traducteurs

Translators' Journal

\title{
L'enseignement du français écrit aux francophones
}

\section{Jacqueline Bossé-Andrieu}

Volume 27, numéro 3, septembre 1982

URI : https://id.erudit.org/iderudit/001889ar

DOI : https://doi.org/10.7202/001889ar

Aller au sommaire du numéro

Éditeur(s)

Les Presses de l'Université de Montréal

ISSN

0026-0452 (imprimé)

1492-1421 (numérique)

Découvrir la revue

Citer cette note

Bossé-Andrieu, J. (1982). L'enseignement du français écrit aux francophones. Meta, 27(3), 342-347. https://doi.org/10.7202/001889ar d'utilisation que vous pouvez consulter en ligne.

https://apropos.erudit.org/fr/usagers/politique-dutilisation/ 
BLOC-NOTES

\section{L'ENSEIGNEMENT DU FRANCAIS ÉCRIT AUX FRANCOPHONES*}

Il me faut tout d'abord préciser ce que j'entends par cours de «rédaction». En fait, je donne à ce terme un sens très général et je regroupe, sous cette rubrique, tous les cours visant au perfectionnement de la connaissance de la langue maternelle, langue vers laquelle les étudiants sont en principe appelés à traduire. J'ajoute encore que je me limiterai au domaine qui m'est familier, c'est-à-dire l'enseignement du français aux francophones... Toute une gamme de cours seront envisagés, depuis les cours que l'on hésite à appeler cours de rattrapage, jusqu'aux cours de contraction de textes et de rédaction professionnelle, en passant par les cours de polissage du style.

La plupart des écoles européennes et canadiennes astreignent leurs étudiants à des cours de français. Un exemple précis et éloquent: l'École d'interprètes internationaux de Mons impose, en première année, 75 heures de cours intitulés «Langue de base et expression de la pensée» et, en deuxième année, les mêmes cours se poursuivent à raison de 120 heures. Il faut cependant spócifier que les horaires, en Europe, sont beaucoup plus chargés qu'ici et comportent, en moyenne, 35 heures de cours par semaine. Au Canada, si les écoles tendent à enseigner de plus en plus la rédaction professionnelle, par contre les cours de perfectionnement proprement dit n'y sont pas toujours de règle. Les exigences des universités dont les écoles canadiennes dépendent expliquent sans doute cet état de choses. Néanmoins, quand on examine les programmes des écoles canadiennes ou européennes, force est de constater que, dans tous, la rédaction est mise au service de la traduction.

I-Liens, entre la traduction et la rédaction; les raisons d'enseigner la rédaction aux futurs traducteurs.

* Colloque de Glendon, 1980.
Personne ne niera les liens qui unissent traduction et rédaction. C'est un truisme de dire que le traducteur est avant tout un rédacteur. Le guide de l'étudiant de l'École de traducteurs et interprètes de Genève définit ainsi la tâche du traducteur:

La tâche du traducteur est de restituer par écrit et sous une forme "achevée» un texte rédigé dans une autre langue. Pour ce faire, il doit non seulement connaitre à fond la langue du texte original, mais aussi - et surtout - celle dans laquelle il traduit. Aussi doit-il être exercé à l'analyse de textes, posséder le sens du style et maâtriser les moyens d'expression. Le futur traducteur doit également perfectionner son vocabulaire pour se rendre plus indépendant des outils auxiliaires (dictionnaires et lexiques) afin de trouver quasi spontanément l'expression, le terme ou la locution appropriée ${ }^{1}$.

L'avis des «professionnels» de la traduction n'est point différent. Dans une communication présentée à la huitième Biennale de la langue française, M. Jacques Flamand, chef-traducteur et rédacteur au Conseil des Arts du Canada, et professeur à temps partiel de rédaction à l'ETI d'Ottawa, affirmait, dans son analyse des rapports théoriques entre traduction et rédaction:

Tout traducteur et, a fortiori, tout réviseur doit avoir un talent de rédacteur [...] La rédaction est partie intégrante de l'acte de traduire et la formation du traducteur ne saurait se concevoir sans un apprentissage poussé de la rédaction. Traduire, n'est-ce pas une forme de rédaction ${ }^{2}$ ?

Et quelques pages plus loin :

Les techniques de la rédaction et le maniement du langage doivent être à la base de l'apprentissage de la traduction ${ }^{3}$.

En outre, des liens très pratiques unissent, au Canada, traduction et rédaction. On le sait, un fort mouvement « rédactionnel » se fait sentir au Canada depuis quelques années. Certains soutien-

1. Guide de l'étudiant de l'ETI de Genève, p. 2.

2. Jacques Flamand (1980): "Traduction et rédaction. Leurs rapports dans la situation canadienne actuelle», conférence prononcée à la $8^{\mathrm{e}}$ Biennale de la langue française, janvier, p. 3.

3. Ibid., p. 5 . 
nent toutefois que les besoins en traductionrédaction sont fortement exagérés. Quoi qu'il en soit, plusieurs d'entre vous se souviennent peut-être de la communication de Jacques Poisson dans laquelle, au huitième congrès mondial de la Fédération internationale des traducteurs (Montréal, mai 1977), le conférencier demandait que cesse la distinction entre la profession de traducteur et celle de rédacteur et que les écoles de traducteurs insistent davantage sur l'enseignement de la rédaction.

lLe même été, au Québec, l'adoption de la Charte de la langue française qui faisait du français la langue des communications institutionnalisées, de l'administration gouvernementale, de la justice, de l'enseignement, a donné encore plus d'élan à ce mouvement "rédactionnel " et a entraîné une diminution du volume des traductions et une augmentation de celui des rédactions. À partir de cette époque, les programmes de certaines écoles de traduction canadiennes incluent des cours de rédaction professionnelle.

Parallèlement, les services linguistiques des entreprises privées se mettent à recruter un nornbre croissant de rédacteurs, confirmant ainsi les propos tenus, en 1975, par M. Raymond Frenette, alors directeur des services linguistiques de la Sun Life et vice-président de la Société des traducteurs du Québec, qui prédisait que «le service de traduction [allait] devenir un centre de rayonnement du français dans l'entreprise» et que, «de plus en plus on [ferait] de la rédaction parallèle au lieu d'une simple traduction ${ }^{4}$. Ainsi, la Société d'énergie de la baie Jarnes, par exemple, engage des rédacteurstraducteurs qui doivent pouvoir rédiger, corriger et traduire. L'examen écrit, conçu par le Service «Rédaction et Traduction ", comprend, en général, une courte traduction, une épreuve de révision (sans que soit fourni le texte de départ), et le commentaire d'une expression fautive. Il ressort clairement que l'accent est mis sur les aptitudes à rédiger - insistance qui n'a rien d'étonnant si l'on sait que les principales fonctions du Service de Rédaction et de Traduction de la Société d'énergie de la baie James sont:

- réviser la qualité de textes déjà rédigés en français;

- conseiller les cadres dans la rédaction de certains textes:

- établir des normes de qualité du français, selon la nature des documents

- normaliser et diffuser une terminologie acceptée pour l'ensemble de la SEBJ

- traduire des textes vers l'anglais lorsque certains documents [sont] requis dans les deux langues, et traduire vers le français, au besoin, des textes provenant de l'extérieur's

4. "Table ronde surl'enseignement de la traduction " Meta, vol. 20, $\mathrm{n}^{\circ} \mathbf{I}$, mars 1975 , p. 51

5. Extrait de «La situation linguistique à la so-
Il faut préciser que, selon les termes de Mlle Sansregret, chef des services linguistiques, depuis 1973, année de l'adoption d'un règlement intérieur sur la langue de travail, la SEBJ n'est pas «une entreprise à franciser, ni même une entreprise francisée, mais bien une entreprise fonctionnant en français $* 6$.

On voit, d'après cet exemple, que les fonctions d'un tel service débordent largement le cadre de la traduction. Le phénomène n'est pas isolé puisque, dans maintes entreprises québécoises, le service de traduction, rebaptisé «service(s) linguistique(s)», est appelé non seulement à traduire, à réviser, mais encore à

donner des conseils d'ordre syntaxique, stylistique et terminologique. [...] Dépassant ce rôle de conseil, le service exerce, dans beaucoup de cas, une espèce de direction linguistique, bien que son autorité soit surtout morale $[. .$.$] Tout de même, ses$ attributions le définissent souvent comme l'autorité quant à la norme linguistique [...] Malgré des obstacles pratiques et des échecs particuliers qu'on peut espérer momentanés, il demeure que le Service est investi d'un rôle de normalisation et de surveillance de la qualité du français dans l'entreprise, fonction qui peut ajouter une dimension plus stimulante à son travail ${ }^{7}$.

Pour illustrer cette nouvelle dimension qu'acquièrent les services linguistiques, mentionnons que l'action de certains d'entre eux se fait sentir à l'extérieur de l'entreprise; on peut penser, en particulier, à différentes publications émanant de tels services; par exemple, C'està-dire, publié par le Comité de linguistique de Radio-Canada, les Mots dits grands maux, publié par la SEBJ, et le Guide pratique de correspondance et rédaction, conçu par Brigitte Van Coillie-Tremblay de la Régie des rentes du Québec.

Il convient enfin de dire un mot de la responsabilité du traducteur - et du rédacteur - visà-vis de la langue et de ses concitoyens. En effet, dans un pays bilingue comme le nôtre, l'importance du nombre de textes traduits influe sur la qualité de la langue parlée et écrite. Tout récemment encore, en octobre, au colloque sur la qualité de la langue française, le juge Robert Auclair dénonçait les anglicismes et les fautes de français que comporte la langue des lois et des règlements; prenant des exemples dans des textes qu'il lui avait été donné de lire en sa qualité de fonctionnaire ou de citoyen, il faisait remarquer que les Québécois vivant «dans un bain de traduction» ont tendance à «s'exprimer fréquemment en fonction

ciété d'énergie de la baie James», communication présentée par Louise Sansregret au Colloque sur la qualité de la langue après la loi 101, organisé, à Québec, par le Conseil de la langue française, p. 8 .

6. Ibid., p. 7

7. Gilles Colpron (1976) : «Les fonctions du service de traduction $"$ Meta, vol. $21, \mathrm{n}^{\circ} 1$, mars, p. 66. 
de la langue qu'ils traduisent, l'anglais leur servant de modèle, de maître à parler et cela, évidemment, sans qu'ils en soient conscients ${ }^{8}$.

L'on est en droit de penser qu'avec l'adoption de la Charte de la langue française, la francisation des entreprises au Québec et dans d'autres provinces, et donc l'importance donnée à la rédaction parallèle, les dangers iront en s'amenuisant. Mais encore faut-il que les traducteurs-rédacteurs se montrent à la hauteur du rôle qui leur est dévolu et manient avec une certaine aisance la langue d'arrivée. Or, en ce qui concerne les écoles de traduction, si elles font de leur mieux pour admettre des candidats qui semblent posséder des bases linguistiques assez solides, il leur est impossible de se faire des illusions. Elles doivent reconnaître que la plupart des aspirants-traducteurs qui leur arrivent sont mal préparés, bien que ces derniers soient souvent convaincus qu'il suffit de parler depuis la naissance la langue vers laquelle ils traduiront pour être en mesure de traduire correctement. Beaucoup pensent, à tort, posséder les connaissances linguistiques nécessaires à la pratique de la traduction. Malheureusement, il n'en est rien et l'écart qui sépare langue écrite et langue parlée semble être dans une grande mesure à l'origine des difficultés qu'ils éprouvent. En effet, au cours des études secondaires - ou post-secondaires au Québec - l'accent a été mis sur la langue parlée et sur l'expression de la créativité et de la spontanéité, au détriment de l'apprentissage de la langue écrite. On se souvient à ce propos des articles de la journaliste Lysiane Gagnon, publiés dans la Presse du 5 au 12 avril 1975, et qui ont été réunis dans un recueil intitulé le Drame de l'enseignement du français. Conséquence de cet enseignement : nos étudiants nourrissent trop d'illusions sur la qualité de leur français écrit et sont consternés en constatant le nombre de leurs lacunes; ils avouent que leur apprentissage de la grammaire, de l'orthographe, des lois de l'expression écrite remonte à plusieurs années et le déplorent profondément. Beaucoup écrivent comme ils parlent et comme ils le faisaient avant d'entrer à l'université. Et pourtant, même les écrivains conviennent qu'il est difficile d'écrire. J'ai, tout récemment encore, entendu Jean-Paul Sartre, dans une entrevue rediffusée le lendemain de sa mort par Radio-Canada affirmer que, étant donné l'écart entre le français parlé et le français écrit, quand on écrit, on écrit dans une «langue étrangère ». Et il citait l'exemple d'un ami qui était extraordinaire quand il parlait, mais «lamentable» quand il écrivait.

8. Le juge Robert Auclair, «La langue des lois et des règlements ", communication présentée au Colloque sur la qualité de la langue après la loi 101, organisé par le Conseil de la langue française, p. 23.
$\mathrm{Ne}$ sait donc pas rédiger qui veut et, dans les écoles de traduction, nous ne pouvons que répéter aux futurs traducteurs que l'apprentissage de la rédaction est difficile et que, «celui qui parle le français doit peiner longtemps avant de savoir l'écrire ${ }^{9}$.

$\mathrm{Si}$ les difficultés que doivent surmonter les francophones sont grandes, elles sont insurmontables pour ceux qui se piquent de bilinguisme. Dans leur cas, à part quelques rares exceptions, c'est le plus souvent la catastrophe. Alors que ces étudiants soi-disant bilingues $s$ 'imaginent représenter des candidats idéals, ils éprouvent une amère déconvenue quand il leur faut s'apercevoir qu'ils ne peuvent traduire ni dans une langue ni dans l'autre, et que leur «bilinguisme», au lieu d'être un atout, constitue un obstacle, car «il n'y a, en fait, que quelques virtuoses qui soient capables de manier deux ou plus de deux langues sans que se produisent jamais chez eux les phénomènes qu'on désigne sous le nom d'interférence linguistique ${ }^{10}$.

\section{II - Enseignement de la «rédaction».}

Il est évident, d'après ce que nous venons de dire, qu'un travail important échoit aux professeurs de rédaction, et bien entendu aux professeurs de traduction qui, dans les devoirs d'étudiants, doivent corriger une multitude de fautes de français.

Pour étayer cette affirmation et vous donner un aperçu des points sur lesquels doivent porter les cours de «rédaction» de base, afin d'alléger le travail du professeur de traduction proprement dite, je rapporterai les résultats d'une expérience tentée à l'ETI de l'Université d'Ottawa. L'École avait organisé une «journée de traduction» avec la collaboration du gouvernement fédéral. C'est-à-dire que, un jour, de neuf heures à cinq heures, des étudiants volontaires de troisième année ( $2 \mathrm{e}$ année de traduction) ont traduit un texte choisi et corrigé par des réviseurs de la Fonction publique. (Certains d'entre vous ont sans doute lu avec intérêt le compte rendu, rédigé par Daniel Gouadec pour le numéro 24 de Meta, de séances analogues qui avaient eu lieu en 1978-79). Une semaine plus tard, les réviseurs sont venus à l'École rendre les copies et apporter des commentaires à la révision. Finalement, les critiques les plus nombreuses adressées aux étudiants concernaient, non pas des fautes de traduction proprement dite, mais des fautes de français. Par exemple, il leur a été reproché de ne pas connaître le sens exact des mots, (confusion entre «exploitation» et «extraction», des étudiants ayant écrit: «extraction de mines»),

9. André Martinet (1974): «Les chances du français", Le français sans fard, Paris, P.U.F., p. 23

10. Id., Éléments de linguistique générale, Paris, Armand Colin, p. 169. 
d'avoir placé des infinitifs en anacoluthe (ex: «pour pouvoir enfouir les câbles de transmissions, les conducteurs doivent être isolés»), d'avoir laissé des pléonasmes et des formules redondantes («promettre pour l'avenir», «des mesures correctives pour remédier à la situation»), d'avoir employé des verbes incolores (avoir, être, faire, mettre), d'avoir commis des solécismes, et enfin des fautes de style (cascade de "de $"$ ), sans compter les fautes d'orthographe et de grammaire.

$A$ propos, d'ailleurs, d'une remarque faite par l'un des réviseurs m'est revenue à l'esprit une question que je me pose depuis longtemps: quel français devons-nous enseigner dans les écoles de traduction, ou encore, quelle attitude devons-nous adopter vis-à-vis du français? Je m'explique: un réviseur a relevé, dans des copies, les conjonctions "quoique» et «malgré que» et a mis en garde les étudiants contre ces deux formules en leur citant Jacques Cellard selon qui, "quoique» "manquant d'élégance" et «malgré que» étant condamné par l'Académie, il vaut mieux, dans les deux cas, s'abstenir et employer, si on ne peut faire autrement, «bien que», qui présente toutefois l'inconvénient d'être bien lourd lui aussi. Donc, quand vous enseignez des cours de rédaction, devezvous vous montrer puriste ou laxiste? Dans le premier volume de la Grammaire française, Léon Robert Wagner, bien que n'ayant pas à l'esprit la formation de spécialistes de la langue, donne à la question posée plus haut la réponse que nous cherchions :

Le rôle des maitres est [...] de proposer comme les meilleures les normes auxquelles se plient la majorité des écrivains, et d'enseigner au public, en dehors d'un purisme sectaire, à s'y soumettre, comme eux, selon les circonstances. Le purisme sectaire conduit au ridicule, à l'artifice. L'attitude contraire, si on la favorisait d'une manière systématique, aboutirait à un résultat encore plus déplora-

ble, puisqu'à la longue il interromprait la transmission d'une culture "1.

Par conséquent, si le rôle du professeur de rédaction est, à mon avis, essentiellement de conseiller l'étudiant, de lui indiquer quelles sont les formes admises ou critiquées, et pour quelles raisons, il lui faut bien, par la force des choses, enseigner la norme - ou les normes - d'autant plus que certains étudiants travailleront pour des réviseurs puristes à l'extrême. Ainsi, une fois sur le marché du travail, les étudiants qui auront reçu une telle formation seront-ils en mesure de se défendre et de comprendre les remarques faites par leur supérieur hiérarchique, et, éventuellement, de savoir pourquoi un réviseur admet les formes critiquées par un autre.

Le professeur de rédaction doit donc être une source de renseignements; il lui incombe,

11. Léon Robert Wagner (1973) : la Grammaire française, I, Paris, SEDES, p. 51. en outre, d'apprendre aux étudiants à réfléchir sur leur langue et à résoudre les difficultés qu'ils rencontrent dans la pratique de l'écrit. Et le champ d'action est immense, même lorsque l'étude de la grammaire ne s'avère pas nécessaire. Tout d'abord, l'un des points auxquels il faut absolument s'attaquer est celui des anglicismes et des régionalismes, sans pour autant condamner systématiquement ces derniers. Dans le cas des anglicismes, il me semble indispensable de faire prendre conscience aux étudiants des risques d'interférences, de les habituer à exercer leur sens critique et à déceler les anglicismes contenus dans des textes de tout genre, y compris les leurs, de leur fournir les raisons, qu'ils n'entrevoient pas toujours, pour lesquelles un terme constitue, dans une phrase donnée, un anglicisme, et enfin, et surtout, de leur montrer l'emploi correct du même terme. En effet, trop d'étudiants dont le vocabulaire ne brille déjà pas par la richesse, ont tendance, lorsqu'ils se méfient d'un terme, à l'éliminer complètement de leur langue. Et c'est là l'erreur dans laquelle il ne faut pas verser.

En second lieu, l'accent devrait porter sur la précision lexicale, car le traducteur n'a pas le droit d'ignorer le sens précis des mots qu'il emploie même si celui de certains mots n'est pas clair pour beaucoup d'entre nous. Permettezmoi de rappeler ici une expérience menée par Jean Guénot et rapportée dans les Clefs pour les langues vivantes: l'auteur a interrogé cinquante personnes sur le sens précis de «glabre» et de "glauque». Résultats: vingt-huit personnes sur 50 ont attribué à ces deux mots le sens que donne le dictionnaire. Mais, pour d'autres, «glauque » signifiait luisant, ou gluant, ou écceurant, ou humide (alors qu'il signifie vert), et, pour «glabre» (dépourvu de poil), certains ont répondu maigre ou pâle ou sévère ou malade. Jean Guénot conclut :

La majorité des gens interrogés sont fiers de connaître leur langue; les contraindre à admettre qu'ils ne la connaissent pas entièrement est une contrariété visible. Habituez-vous donc à savoir que vous ne connaissez pas entièrement votre langue; personne ne la connait entièrement ${ }^{12}$.

Mais il faut ajouter, à l'adresse de nos étudiants, qui ne sont nullement à l'abri de semblables défaillances, qu'il est indispensable que eux la connaissent aussi bien que possible, car, «ce que le citoyen ordinaire ne sait pas ou n'emploie guère puisque rien ne l'y oblige, nous n'avons pas le droit, nous, [traducteurs] de l'ignorer ${ }^{13}$. Pour les aider dans cette voie, il faut les exercer à trouver le mot juste, à saisir les nuances qui séparent des mots de

\footnotetext{
12. Jean Guénot (1974): Clefs pour langues vivantes, Paris, Séghers, p. 171.

13. Irène de Buisseret (1975): Deux langues, six idiomes, Ottawa, Carlton-Green, p. 384.
} 
prime abord synonymes, à savoir par exemple que tel verbe s'emploie avec tel substantif (en les mettant toutefois en garde contre l'abus des clichés), etc.

Une fois ces étapes franchies, il parait utile, pour affiner la sensibilité linguistique de nos futurs traducteurs, de les faire «jouer» avec l'expressivité. Il est intéressant, à ce propos, de mentionner que l'examen d'entrée à l'École d'interprètes (et de traducteurs) de Mons accorde une grande importance à des questions dans lesquelles les candidats doivent classer des phrases selon leur expressivité. En restant toujours dans la stylistique interne, il est primordial d'enseigner à écrire en variant les niveaux et les registres de langue et de montrer comment un même message s'exprime différemment selon le locuteur ou le destinataire. Une anecdote, citée par Sven Sainderichin et empruntée aux Techniques de l'exposé et des communications orales de Roger P. Wilcox illustre l'importance qu'il y a à rédiger - ou à traduire - selon le destinataire : c'est l'histoire d'un plombier qui demande à un service de documentation si l'acide chlorhydrique est un bon produit à utiliser pour déboucher les tuyaux. Il lui est répondu: «L'efficacité de l'acide chlorhydrique est indiscutable, mais le résidu corrosif que provoque ce produit est incompatible avec la permanence du métal». Et notre plombier, tout heureux, remercie le service de lui avoir confirmé l'efficacité de l'acide en question. Ce n'est qu'après plusieurs échanges épistolaires que, finalement, le patron du service prend la plume pour écrire au plombier: «N'employez pas l'acide chlorhydrique. Ça ronge les tuyaux $»^{14}$.

Les étudiants n'éprouvent, en général, après quelque temps, que peu de difficulté à reconnaître des termes ou des expressions appartenant à un registre spécialisé. Mais ils semblent avoir plus de mal avec les niveaux de langue. Ainsi, une de mes étudiantes, dans la description d'une pièce repoussante de saleté (devoir qui nécessitait l'emploi de termes précis, péjoratifs et évocateurs), avait écrit: «Les couleurs les plus dégueulatoires s'offraient à ma vue». Le contraste entre les deux parties de la phrase, entre le terme vulgaire et le cliché pseudolittéraire, est sans aucun doute peu heureux c'est un euphémisme - , mais quelle n'a pas été ma surprise d'entendre l'étudiante répliquer, d'un ton véhément : "Comment, dégueulatoire, un terme vulgaire?» Et d'ajouter : «Pour moi, c'est un mot élégant, recherché, qui fait bien». Pour cette étudiante, qui, par rapport aux autres, connaît bien le français, lit beaucoup et déploie de grands efforts pour écrire en français «international " sinon en «français de

14. Sven Sainderichin (1976) : Ecrire pour être $u$, Paris, Entreprises modernes d'édition, p. 20.
France», le mot «dégueulatoire» était un mot prestigieux. On remarque ici le désir de se conformer à la langue d'une élite, phénomène qui s'apparente à celui des faits de mode décrits par Charles Bally dans le Langage et la vie:

La mode est, psychologiquement, un phénomène complexe. A sa base, il y a d'abord un désir (donc tout le contraire d'une contrainte), désir de se distinguer de son milieu en adoptant les caractères ou les signes extérieurs d'un groupe jugé supérieur [...] La mode n'épargne pas le langage et l'attaque de plusieurs manières ${ }^{15}$.

Une autre observation relative aux niveaux de langue est l'incapacité qu'éprouvent d'autres étudiants à se dégager du style familier. Certains n'écrivent bien qu'en employant le langage de la conversation, celui de tous les jours, conséquence, sans doute, de l'enseignement du français au CEGEP. Lysiane Gagnon, que j'ai déjà citée, a dénoncé un système scolaire dans lequel les enseignants ne talonnent pas les élèves pour ne pas briser leur spontanéité, bien que ces derniers ne «réussissent pas à acquérir un niveau de langue autre que celui de la langue familière ${ }^{16}{ }^{16}$.

Il faut aussi, à mon avis, habituer les étudiants à tenir compte des connotations péjoratives - ou mélioratives - de certains mots. Pour vous donner un exemple de l'emploi fautif d'un terme dont l'utilisateur ignorait manifestement la nuance péjorative - exemple que vous avez peut-être vous aussi relevé - citons le journal télévisé de Radio-Canada, qui, plusieurs fois au cours des semaines qui ont précédé le référendum, a annoncé avec le plus beau sérieux que $M$. $X$, accompagné, dans un cas, d'une "brochette» de députés fédéraux, s'était adressé à la population de telle ville. Bien sûr, les dictionnaires ne sont pas toujours aussi complets qu'on le voudrait. Ainsi, pour «une brochette de», le Lexis donne simplement comme définition: «un petit groupe de»; cependant, l"exemple qui suit est éloquent: «Il y avait une belle brochette de notabilités ventrues et épanouies au premier rang de l'assistance». (Notons en passant que le Petit Robert 1978 ne mentionne que le sens propre de «petite broche servant à porter sur l'habit plusieurs médailles ou décorations : cette série $\%$ ).

Ce sont donc des nuances de ce genre qu'il est bon d'accoutumer les futurs traducteurs à saisir. Bien entendu, l'enseignement de la rédaction doit viser, non seulement à la correction syntaxique et à la précision lexicale, mais aussi à la simplicité, à la concision et, si possible, à l'élégance du style. Un exercice est, à cet égard, extrêmement formateur: c'est celui de la contraction de textes, que presque

15. Charles Bally, le Langage et la vie, Genève, Librairie Droz, p. 126.

16. Lysiane Gagnon (1975) : le Drame de l'enseignement du français, Montréal, La Presse, p. 23. 
tous les programmes de traduction incluent. Qu'il soit appelé compte rendu, résumé ou contraction de texte, le principe en demeure le mêrne: restituer, sous une forme condensée, un texte écrit par un autre. Les étudiants ne sont donc pas astreints à "se creuser la tête " pour trouver des idées, et tous leurs efforts peuvent porter sur l'agencement des idées données et sur la correction de l'expression. Les affinités de cet exercice avec la traduction sont évidentes, comme le fait observer Jean-Marie Gautherot, professeur chargé, en 1972, des questions pédagogiques aux Services culturels de l'Ambassade de France à Vienne:

Visant une restitution «substantifique» et non exhaustive d'un contenu de signification, la contraction mobilise la compétence linguistique de l'opêrateur sur une aire plus élargie en même temps que plus touffue. Non qu'elle fasse bon marché de la précision, mais elle exige un inventaire plus étendu des champs lexico-sémantiques dans lesquels sont choisies les unités propres à exprimer une signification. Reconstruisant d'autre part sur un texte de base un texte second, elle implique une nouvelle hiérarchisation des éléments et, partant, une cohérence nouvelle du discours. Aussi conçoit-on aisément non seulement qu'elle puisse occuper à côté de la traduction une place complémentaire de plein droit (elle serait à la traduction, mutatis mutandis, ce que la lecture rapide est à la lecture explication), mais aussi qu'elle représente, comme celle-là, et à un niveau défini, un instrument pédagogique utile de perfectionnement linguistique ${ }^{17}$

Avec cet exercice, nous faisons alors d'une pierre deux coups - ou même trois coups si l'on considère que l'entraînement à la contraction de textes contribue aussi à faire acquérir un esprit de synthèse qui fait très souvent défaut à nos futurs traducteurs et dont il se pourrait qu'ils aient besoin dans leur carrière C'est ainsi que le rapport qu'il m'a été donné de lire sur la visite officielle effectuée, en mars 1979, par l'ETI de Genève aux services linguistiques de la Communauté européenne signale que les traducteurs des communautés européennes doivent non seulement traduire et faire du «rewriting" (puisque «les interventions orales des membres du Parlement sont soumises à une première traduction interne de langue parlée en langue écrite, avant d'être transposées dans les cinq autres langues»), mais que parfois il leur faut aussi rédiger «des résumés de documents de travail dont seuls certains passages seront ensuite intégralement traduits ${ }^{18}$.

Si l'apprentissage de la contraction de textes figure, comme nous l'avons dit, au plan d'études de la plupart des écoles de traduction

17. Jean-Marie Gautherot (1972) : «La contraction de textes", Le français dans le monde, juin, p. 30

18. «Rapport sur la visite d'information de l'École de traduction et d'interprétation de Genève aux services linguistiques de la Commission des Communautés européennes », mars 1979, p. 3. européennes et canadiennes, par contre, autant que je sache, ce n'est qu'au Canada qu'est enseignée la rédaction professionnelle, c'est-àdire la rédaction de textes techniques, juridiques, commerciaux, etc. (Montréal et TroisRivières dispensent, par exemple, des cours de rédaction de «documents de nature administrative et commerciale».). Ces cours répondent à des besoins spécifiquement canadiens puisque, comme nous l'avons vu plus haut, à l'heure actuelle, un nombre croissant de textes sont rédigés, au Canada, directement en français. Il arrive que ces cours soient enseignés en relation avec des cours de traduction de textes de même nature et même indépendamment pour former des rédacteurs spécialisés, et nous touchons là à la limite des cours mis, strictement parlant, au service de la traduction.

\section{CONCLUSION}

Ces considérations nous amènent à la conclusion de cet exposé dans lequel je vous ai fait part de quelques vues personnelles sur l'enseignement de la langue de base tel que je le conçois dans une école de traduction. C'est ainsi qu'après avoir constaté qu'il était impossible de dissocier traduction et rédaction pour des raisons inhérentes à l'art de traduire, et aussi pour des raisons dictées par le marché du travail, $\mathrm{j}$ 'ai examiné certains points sur lesquels devraient porter les cours de langue dont le but est de développer les aptitudes à la rédaction et d'apprendre à manier la langue avec une certaine aisance à des étudiants qui n'ont pas eu la chance, comme Maurice-Edgar Coindreau, «le pape des traducteurs», d'apprendre à «lire et à écrire " à l'école secondaire ${ }^{19}$. Ne nous leurrons pas cependant; l'art d'écrire exige de longs et patients efforts: il n'existe pas, dans ce domaine, comme en cuisine, de "recettes faciles». La tâche du professeur de rédaction est, à la fois, ardue et ingrate: il doit se contenter, pendant quelques heures par semaine, de donner à ses étudiants les bases qui leur permettront, à force de travail, d'acquérir les aptitudes à la rédaction indispensables à tout traducteur.

JACQUELINE BOSSÉ-ANDRIEU 